\title{
Optical control of diffuse light storage in an ultracold atomic gas
}

\author{
Leonid V. Gerasimov, ${ }^{1}$ Igor M. Sokolov, ${ }^{1,2}$ Dmitriy V. Kupriyanov, ${ }^{1}$ \\ Rocio G. Olave, ${ }^{3}$ and Mark D. Havey ${ }^{3, *}$ \\ ${ }^{1}$ Department of Theoretical Physics, \\ State Polytechnic University, \\ 195251, St.-Petersburg, Russia \\ ${ }^{2}$ Institute for Analytical Instrumentation, \\ Russian Academy of Sciences, 198103, St.-Petersburg, Russia \\ ${ }^{3}$ Department of Physics, Old Dominion University, Norfolk, VA 23529, USA and \\ *Corresponding author: mhavey@odu.edu
}

We show that coherent multiple light scattering, or diffuse light propagation, in a disordered atomic medium, prepared at ultralow temperatures, can be be effectively delayed in the presence of a strong control field initiating a stimulated Raman process. On a relatively short time scale, when the atomic system can preserve its configuration and effects of atomic motion can be ignored, the scattered signal pulse, diffusely propagating via multiple coherent scattering through the medium, can be stored in the spin subsystem through its stimulated Raman-type conversion into spin coherence. We demonstrate how this mechanism, potentially interesting for developing quantum memories, would work for the example of a coherent light pulse propagating through an alkali-metal atomic vapor under typical conditions attainable in experiments with ultracold atoms.

(c) 2018 Optical Society of America

OCIS codes: $020.1670,270.1670,270.5565,270.5585$

\section{Introduction}

Organization of controllable and lossless light transport through a bulk medium is an important task for applications in quantum information science and for implementation of 
quantum memories in particular, see recent reviews [1, 2] and reference therein. The crucial problem is in identifying those physical systems where transport of a signal light pulse can be reliably delayed, safely stored and retrieved on demand. There are several physical schemes which are considered as candidates for effective light storage. A long-lived quantum memory for a period of a few milliseconds has been recently demonstrated in [3] via Raman scattering on atomic lattice. However because of relatively low overall efficiency such a long-lived atomic memory could be mainly applied to realization of a deterministic single photon source. Other schemes, more oriented toward the problem of a quantum repeater, are based on the $\Lambda$-type conversion of the signal pulse, propagating through a dense atomic system, into the non-decaying spin coherence. That could be done under conditions of either electromagnetically induced transparency (EIT), or a stimulated Raman process, or via a photon echo induced by either controllable reverse inhomogeneous broadening (CRIB) or an atomic frequency comb (AFC). The EIT based scheme in a hot atomic ensemble was successfully demonstrated in [4] with an efficiency of around $40 \%$ and also in [5], where a single photon state was stored in two ensembles of cold atoms with an efficiency of $17 \%$. The echo scheme is preferably implemented in the solid-state systems and proposed to be effective in a multimode configuration. As has been recently reported in [6] the quantum memory based on AFC in an ion-doped crystal can approach an efficiency of around 35\%. At present, the number of reported experiments on atomic memories is increasing rapidly; the reader can find representative references in recent reviews [1, 2].

However, despite obvious progress in developing quantum memories during the last decade the highly effective and long-lived light storage is still a challenging experimental task. For the long distance quantum communication via a quantum repeater protocol [7, 8] the requirements for the reliability of the quantum memories are extremely robust. The memory unit should be highly effective, noiseless and reproducible on a controllable time scale at each segment of the multipath quantum channel. The problem becomes even more subtle if it is addressed to the quantum network operating with continuous variables, see [9, 10]. Any new physical approaches would evidently be desirable for further progress in this challenging and rapidly developing area of quantum information science.

In the present article we establish that coherent multiple scattering, or diffuse light propagation in a disordered atomic medium prepared at ultra-low temperature, represents a promising process for the effective light storage and organization of the quantum memory 
protocol. We emphasis that the process as a whole contains elements of coherent control of light scattering via electromagnetically induced transparency and, at the same time, relies on the coherence of multiple light scattering in an ultracold atomic gas. The main point in this investigation is that the entirety of a propagating signal pulse scattered by the medium is not lost (as is normally estimated in standard schemes based on either electromagnetically induced transparency (EIT) or Raman process for forward propagating light), but transformed into a diffusely propagating, but coherent, mode. This mode can be effectively controlled and stored by initiating its stimulated Raman-type conversion into atomic spin coherence. We theoretically demonstrate how it can be done.

The paper is organized as follows. In section 2 we describe our basic physical idea concerning how the light diffusion can be controlled with coherent light. In this section we also detail our calculation approach. Section 3 presents the results of our numerical simulations and gives the estimates of the delay effect for the light scattered via the Rayleigh scattering channel. Section 4 focuses on the problem of quantum memory and specifically we show one example of how the Bell detection and the proposed quantum memory unit could be integrated in one node of general quantum network. The main results of the paper are emphasized in the conclusions.

\section{Light diffusion controlled via stimulated Raman process}

First consider the situation when a light pulse diffusely propagates through an opaque atomic medium. Here we will ignore in this process effects associated with atomic thermal motion. Such a situation may be approximately obtained for light scattering by an atomic ensemble

prepared in a magneto optical trap (MOT) [11]. Specifically, let us consider a ${ }^{85} \mathrm{Rb}$ sample and a pulse resonant with the closed $F_{0}=3 \rightarrow F=4$ transition associated with the $D_{2}$-line. As shown in Figure 1, the original forward propagating light pulse is split by atomic scatterers into fragments and emerges from the sample more or less isotropically. This process is normally termed incoherent scattering, with the implied emphasis that coherence should be associated with the forward transmitted part of the light. However, any light fragment elastically scattered via a Rayleigh channel in any direction is generated by a coherent response of the atomic polarization to the incident light. Therefore the secondary and multiply scattered waves assemble coherently and eventually create a configuration-dependent emergent spatial mode. Moreover, in an optically dense configuration, only a negligible contribution 


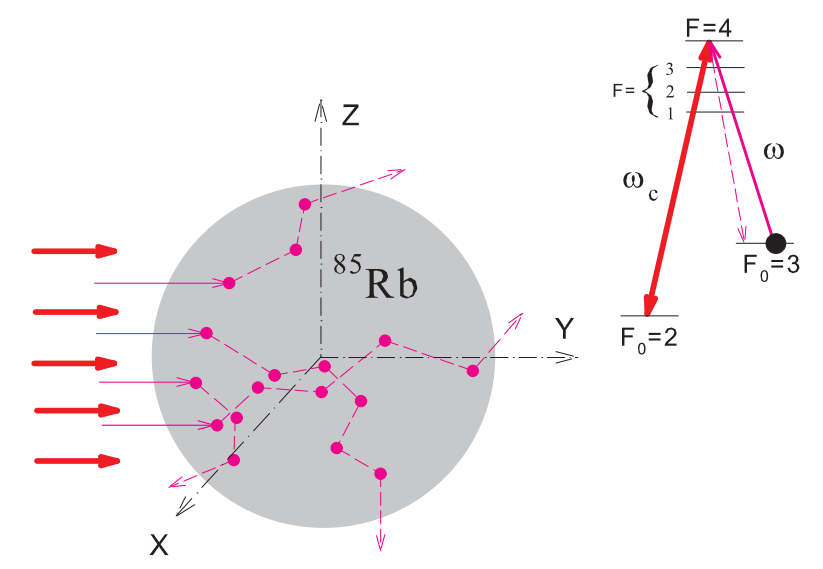

Fig. 1. (Color online) Geometry of the proposed experiment and the excitation scheme, see text. The diffuse propagation of a signal mode of frequency $\omega$ is indicated by pink thin lines and arrows. The diffusion process is affected by a strong control mode of frequency $\omega_{c}$ indicated by red and thick arrows.

of the input light pulse is transmitted by the sample in the forward direction since the free propagation length scale for a resonant photon $l_{0} \sim 1 / n_{0} \sigma_{0}$, where $n_{0}$ is the atomic density and $\sigma_{0}$ denotes the resonant light scattering cross section, is much shorter than the sample length. The diffusion mechanism dominates in dynamics of the light transport in such medium. Since the upper hyperfine sublevels $F=3,2$ are far detuned from the selected excitation channel, spontaneous Raman scattering makes a negligible contribution to the diffusion process.

Let us make a crucial modification of the above process and additionally apply a strong control mode between the lower $F_{0}=2$ and upper $F=4$ hyperfine sublevels. Of itself, this transition is forbidden, but evidently the applied field opens the stimulated Raman channel via far-off-resonance coupling with other hyperfine sublevels of the upper state $F=3,2$. This consists of many Lambda-type couplings strongly interfering in the entire process, see Appendix A for details. Now both the elastic Rayleigh and stimulated Raman processes affect transport of the coherent signal pulse. The influence of Raman scattering is much weaker but its associated transition probability accumulates with each step of a multiple scattering path, that is, while the photon propagates through the sample along a random and zigzag-type diffusion path, see figure 1, The crucial property of the stimulated Raman interaction is that the photon scattered into the control mode will be, with great probability, 
retrieved into the signal mode and we shall discuss how this process affect the signal pulse propagation in more detail.

We restrict our consideration by tracing the dynamics of a weak signal mode when its outgoing amplitude is obtained by linear transformation of its original amplitude in the incident wave. Then the light diffusion can be analyzed via following the transformation of only the first order correlation function of the signal light. This correlation function, being the function of spatial coordinates $\mathbf{r}$ and $\mathbf{r}^{\prime}$ and time arguments $t$ and $t^{\prime}$, is given by

$$
D_{\mu \nu}^{(E)}\left(\mathbf{r}, t ; \mathbf{r}^{\prime}, t^{\prime}\right)=\left\langle E_{\nu}^{(-)}\left(\mathbf{r}^{\prime}, t^{\prime}\right) E_{\mu}^{(+)}(\mathbf{r}, t)\right\rangle
$$

where $E_{\mu}^{(+)}(\mathbf{r}, t)$ and $E_{\nu}^{(-)}\left(\mathbf{r}^{\prime}, t^{\prime}\right)$ are the Heisenberg operators of positive and negative frequency components of the electric field, $\mu$ and $\nu$ are vector indices in the plane orthogonal to the light local propagation direction, and the angle brackets denote the statistical averaging over the initial state of the light and atoms. The total intensity of the scattered light $I(t)$ is expressed by the sum of diagonal components of the correlation function considered at coincident moments of time and integrated over all propagation directions outside the medium.

In a dilute medium, when the mean transport path is long enough such that $l_{0} \gg k^{-1}=\lambda$, for a light ray propagating in any direction there is a preferable coherent enhancement for its current forward propagation. This means that along any ray, for a short mesoscopic scale consisting of a large number of atoms, there is only a slight attenuation of the propagating wave. Such an attenuation comes from the events of random single atom scattering (as was commented above, usually termed incoherent scattering), which have small but not negligible probability. The mesoscopically averaged light propagation is properly described by the macroscopic Maxwell equations and characterized by the sample susceptibility tensor

$$
\chi_{i j}(\omega)=\chi_{0}(\omega) \delta_{i j}+\chi_{i j}^{(A T)}(\omega), \quad i, j=(x, y, z)
$$

which, in our case, consists of a dominant linear isotropic term $\chi_{0}(\omega)$ and a nonlinear anisotropic correction $\chi_{i j}^{(A T)}(\omega)$ associated with the "dressing" of the upper state by the control mode, which is known as the Autler-Townes (AT) effect [12, 13]. The single atom scattering is described by the scattering tensor, which can be qualitatively interpreted as a microscopic susceptibility related to a local inhomogeneity associated with an isolated atomic scatterer. Both the characteristics susceptibility and scattering tensor are subsequently introduced in Appendix A. 
The perturbation theory diagram analysis shows that under the discussed conditions the correlation function of the outgoing probe pulse can be evaluated via summing of socalled "ladder"-type diagrams. The "crossed"-type diagram correction should be added only for evaluation of the backwards scattered radiation [11], which makes, for the conditions considered here, a negligible contribution to the light scattered into the total solid angle. After mesoscopic averaging, the actual light wave in the sample can be visualized as a set of unknown zigzag paths, whose vertices consist of atoms scattering the light from the direction of forward propagation, see Figure 1. Any randomly chosen path contains a chain of atoms located at the vertices and their number is just associated with the scattering order. Such a qualitative physical picture correctly reproduces the entire calculation procedure based on the diagram analysis and on the Monte-Carlo simulation scheme. Each chain of atomic scatterers makes a partial contribution to the formation of the outgoing wave and the total intensity $I(t)$ of the probe pulse emerging the sample can be expanded in the following series

$$
I(t)=\sum_{n=0}^{\infty} I^{(n)}(t)
$$

where each partial contribution $I^{(n)}(t)$ relates to the $n$-th order of multiple scattering. It is important that, as a result of mesoscopic averaging, this series is converging rapidly and only the multiple scattering of relatively low orders (around squared optical depth of the sample) contribute significantly to formation of the pulse intensity or its correlation function (11).

In Figure 2 we reproduce one example of our calculations of the sample susceptibility for a geometry where signal and control modes are in linear but orthogonal polarizations. As is clearly seen from the plotted graphs the dominant contribution comes from the linear term and all three allowed resonance transitions for excitation from the lower $F_{0}=3$ to the upper $F=4,3,2$ hyperfine sublevels of $D_{2}$-line are shown. These resonances have Lorentzian profiles characterized by the atomic natural line width $\gamma$. The nonlinear susceptibility of the medium manifests itself as a narrow but large amplitude spectral feature in the "absorption" (actually associated with the scattering losses) and dispersion profiles near the $F_{0}=3 \rightarrow$ $F=4$ resonance transition. For the calculated AT resonance the amplitude of the control mode is given by the Rabi frequency $\Omega_{c}=2\left|V_{n m^{\prime}}\right|=3 \gamma$, which was chosen for a particular transition between the states $\left|m^{\prime}\right\rangle=\left|F_{0}=2, M_{0}=2\right\rangle$ and $|n\rangle=|F=3, M=2\rangle$, see Appendix A, To tune the AT resonance nearer the maximum of the linear "absorption" 


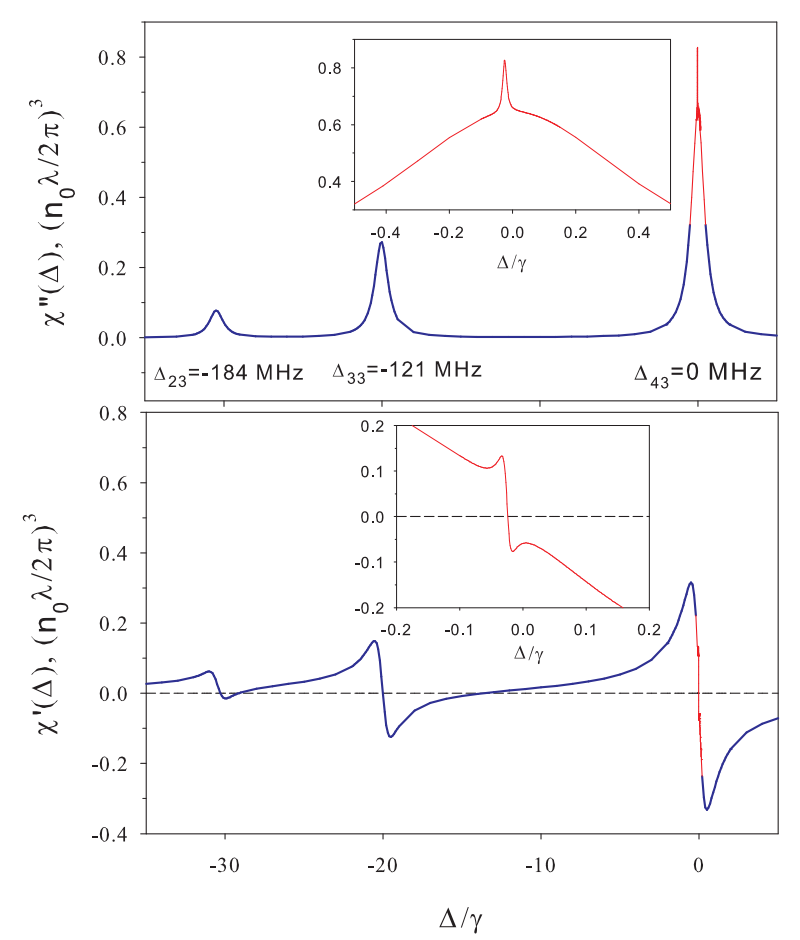

Fig. 2. (Color online) The dielectric susceptibility of the sample $\chi(\Delta)=\chi^{\prime}(\Delta)+i \chi^{\prime \prime}(\Delta)$ as a function of frequency detuning $\Delta=\omega-\omega_{43}$ in units of $n_{0} \lambda^{3}=n_{0}(c / \omega)^{3}$. Upper and lower panels respectively show the absorptive and dispersive components of the susceptibility tensor in the case of linear and orthogonal polarizations of the signal and control modes, see geometry shown in panel (a) of Fig. 3 .

Lorentzian profile the frequency of the control field was slightly shifted from the frequency $\omega_{42}$ such that $\omega_{c}=\omega_{42}-0.4 \gamma$.

The dispersive part of the nonlinear susceptibility governs the stimulated Raman process, which is responsible for an additional delay in the diffuse propagation of the signal pulse. If the pulse spectrum is chosen to be spectrally broader than the AT peak, such that spontaneous Raman scattering is negligible, but still narrower than $\gamma$, then the diffusely propagating light can be effectively delayed for a period of time comparable to or longer than the pulse duration. Roughly speaking, the bandwidth of the AT resonance is scaled as $\Gamma_{\mathrm{AT}} \sim \Omega_{c}^{2} \gamma / \Delta_{\text {hpf }}^{2}$, where $\Delta_{\text {hpf }}$ is the hyperfine splitting in the upper state, such that the required condition $\Gamma_{\mathrm{AT}} \ll \gamma$ is easy to fulfill. In each partial intensity $I^{(n)}(t)$ the electric field amplitude can be approximately expressed by a convolution transform of non-disturbed field, which would propagate in the medium without control mode, and the transfer-type 
function associated with the nonlinear term in Eq.(2) and expressing the action of the control mode. This action consists of the following; along each multiple scattering path the nonlinear coupling with the control mode affects the pulse propagation via its stimulated Raman-type conversion into the spin coherence in the atomic subsystem and recovering into the signal mode with delay. Evidently this effect being applied to the diffusely propagating light would be more effective than in the standard one dimensional geometry associated with only forward propagation. If the control mode is then switched off, this diffusely propagating signal pulse would be stored in the spin coherence. The process of light transport can be regenerated if the control mode is switched on again after a controllable delay time.

\section{Numerical results}

The complete multiple scattering analysis, based on Monte-Carlo numerical simulations, which is evidently required for the protocol demonstration, will be done later and published elsewhere. Here we illustrate the main effects by our calculations done for a single scattering event at the central point of the cloud and at the observation angles coinciding with or orthogonal to the propagation direction of the incident pulse, see geometry and reference frame shown in Figure 1. In our calculations we have assumed a sample generated in a typical MOT configuration characterized by a spherical Gaussian atom distribution with a square variance $r_{0}^{2}$ and peak density $n_{0}$ such that the local density at spatial point $\mathbf{r}$ is given by

$$
n(\mathbf{r})=n_{0} \exp \left(-\frac{\mathbf{r}^{2}}{2 r_{0}^{2}}\right)
$$

The optical depth $b_{0}=\sqrt{2 \pi} \sigma_{0} n_{0} r_{0}$, associated with the customary Beer's law definition, was taken as $b_{0} \sim 10$, which is readily attainable in our laboratory setup. The normalized slow-varying amplitude of the signal pulse $\alpha(t)$ has a Gaussian profile, which spectrum $\alpha(\Delta)$ compared with the AT resonance is shown in panel (a) of figure 3. In the time domain the pulse is parameterized as follows

$$
\alpha(t)=\frac{2}{\left(2 \pi T^{2}\right)^{1 / 4}} \exp \left[-i \Delta t-4 \frac{(t-T)^{2}}{T^{2}}\right]
$$

where $\Delta=\omega-\omega_{43}=0.025 \gamma$ is the small shift between the pulse carrier frequency and the frequency of the atomic $F_{0}=3 \rightarrow F=4$ transition, see Figure 1. This shift allows to optimize the overlap between dispersion part of the AT resonance, calculated in previous section, and the pulse spectrum, see Figure 3(a). The pulse parameters are specified by the 

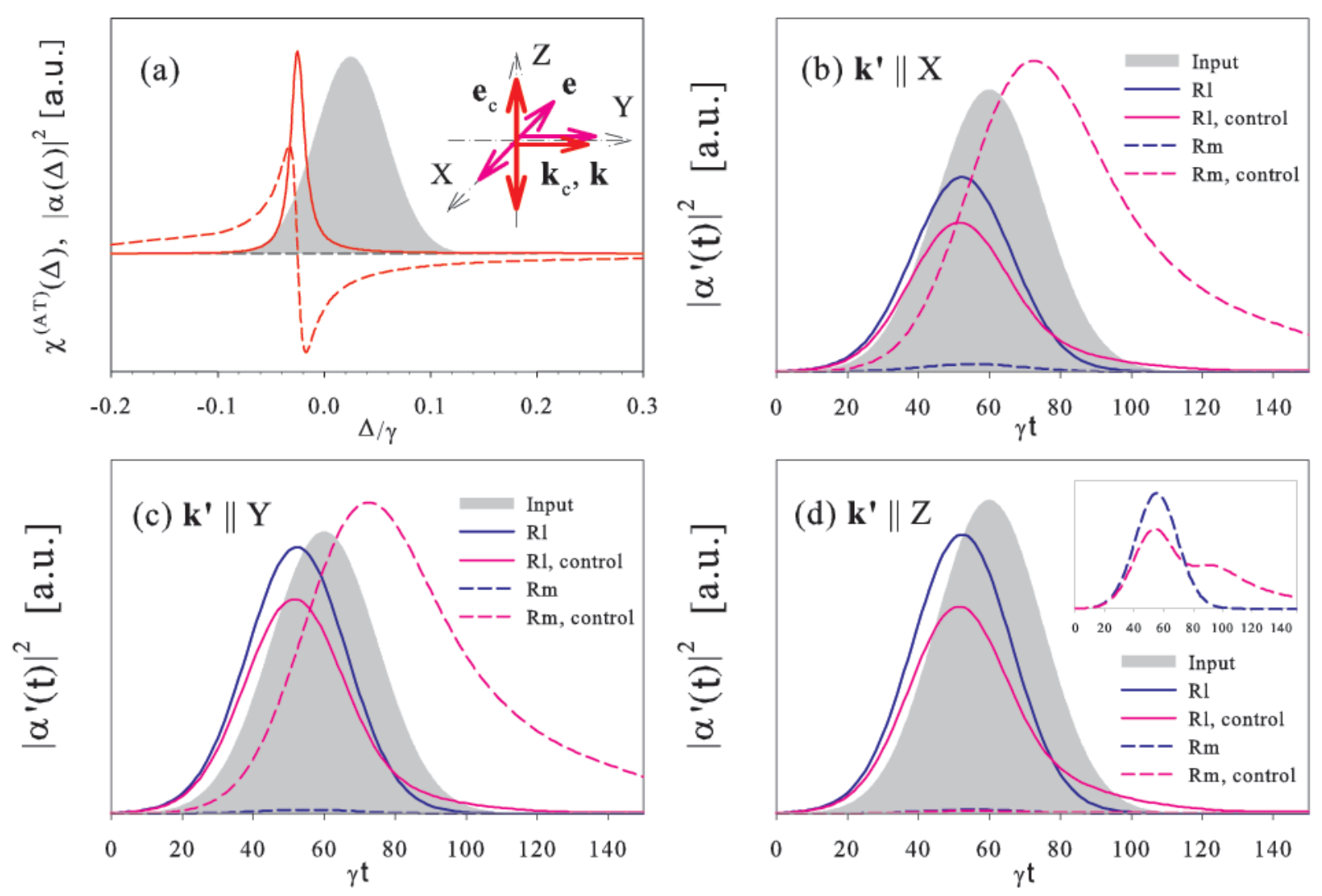

Fig. 3. (Color online) (a): Pulse spectrum vs. Autler-Townes resonance and polarization directions for the control $\mathbf{e}_{c}$ and signal e modes propagating along the $Y$ direction, see Fig. 1. (b)-(d): Fragment of the signal pulse $\alpha^{\prime}(t)$ with wave vector $\mathbf{k}^{\prime}$ generated by single scattering from the central point of the cloud in the directions $X$ (b), $Y$ (c), and $Z$ (d). The blue curves indicate the effect of elastic Rayleigh (Rl, solid) and Raman (Rm, dashed) scattering without the action of the control mode. The pink curves show the corresponding scattering and delay in the presence of the control mode. Comparison of the reference input profile (shadowed) and the scattered light shows superluminal propagation of the scattered light in the spectral domain of anomalous dispersion, see Fig. 2. The delay manifests itself in the long time asymptote of the pulse tail.

mean arrival time $T$ and by the pulse duration (also $\sim T$ ) and determined by one parameter $T=60 \gamma^{-1}$. The spatial distribution of the incoming pulse is considered as flat with a radial aperture $0.5 r_{0}$.

Panels (b),(c), and (d) of Figure 3 respectively show the single scattering response from 
the central point of the atomic cloud (i.e. a single scatterer response) in the direction of $X, Y$ and $Z$ axes such that the optical path in the elastic channel is equal to $b_{0}$ for all the plotted graphs. We consider both the elastic and inelastic scattering channels and extend the definition of Rayleigh scattering by also including the contribution of elastic Raman process in it. It seems natural for our discussion to refer to the entire elastic channel as Rayleigh and inelastic as Raman since all the light scattered elastically undergoes diffusive transport and can be delayed and stored under the protocol described in the previous section. The stimulated Raman process governing this protocol, affects the system dynamically and a certain leading edge of the light pulse will leak because in the beginning stage this dynamical action is always weak. The delay effect is seen most clearly in the long time asymptote of the pulse tale when the stimulated Raman interaction has enough duration to be more fully manifested. This effect is clearly seen in the plotted dependencies. The leakage could be made smaller if the Rabi frequency of the control was higher, but in this case more losses from spontaneous Raman scattering would be expected. For each particular optical depth $b_{0}$ there is an optimal balance between pulse duration and Rabi frequency.

Even in an optimal situation the light scattered from the sample via spontaneous (not stimulated) Raman process generates losses of the signal pulse and induces noise (extra non-informative light) at the retrieval stage of the protocol. As can be seen from the dependencies presented in Figure 3, in the absence of the control mode, the spontaneous Raman channel makes a fully negligible contribution. To estimate the relative amount of the Raman contribution in the single scattering event one should pay attention that for the presented data, in accordance with the Beer's law, the intensity of the Rayleigh fraction is attenuated by the factor $e^{-5} \sim 0.007$ from its amount at the central point of the atomic cloud where it was generated. But as follows from the presented data the Raman fraction, which is not attenuated and propagates to the detector freely, reveals a much smaller contribution than the Rayleigh part. In the presence of the control mode the Raman fraction is significantly enhanced for the scattering in the directions of $X$ and $Y$ axes and becomes even greater than the Rayleigh part. That is a direct consequence of the AT effect and caused by the essential overlap of the pulse spectrum with the "absorption" part of the AT resonance, see Figure 3(a). But again, if one takes into account that the Rayleigh contribution is actually $e^{5} \sim 150$ times greater, then it will become clear that the losses via the spontaneous Raman channel represents still a negligible effect. 
As was indicated in the beginning of this section, the relative amount of spontaneous Raman losses would be most realistically estimated via a complete Monte-Carlo simulation for the scattering into the overall solid angle. That would be given by the sum (3) and include all orders of multiple scattering. But let us point out that the spontaneous contribution is expected to be generally small because of the small overlap of the pulse spectrum with the AT resonance, as is is shown in the first panel of Figure 3. In an ideal situation we expect that there would be low and negligible probability for such an incoherent transition initiated by spontaneous Raman scattering.

It is important that the delay effect, for light emerging the sample via the Rayleigh channel, can be observed from the dependencies of Figure 3. The incoming pulse, with an intensity reduced by a factor $3 \cdot 10^{-10}$ (recall that the plotted data relate to the contribution of a single atomic scatterer), is shown here as a shadowed reference profile. In the case of

only a single scattering event the delay of the scattered fragment, shown in panels (b)-(d) of Figure 3, is partially masked by a superluminal effect caused by anomalous dispersion, but clearly seen in the long time asymptote of the pulse tail. Let us stress that in these graphs the delay should be associated with the scattered fragment and not referred to the original pulse. The superluminal effect is only a manifestation of the fact that the leading edge of the incident pulse mainly contributes to the single scattering signal. The main important point is that if we accumulate all the contributions of a multiple scattering chain (see Fig. 1), the delay can be enhanced by the factor of $b_{0}$ while scaling the length of a real random path for the signal photon in units of its mean straight line path $b_{0} l_{0}$. We emphasize here that the contribution of the Raman channel is expected to be small in each scattering order. For some scattering angles it is even negligible, as is shown in the inset of panel (d) of Figure 3 for scattering in the $Z$-direction, where the Raman signal (not attenuated) has been increased by a scale factor of 100 .

\section{Application to the quantum memory}

Let us briefly discuss how the results of the previous sections could be applied to the quantum memory problem. The main inconvenience of the proposed scheme is that it transforms the signal pulse into its scattered fragments distributed over a substantial solid angle. However, we emphasize that this should not be considered a fundamental barrier for further applicability of the discussed mechanism in a quantum memory protocol. In principle the 
light scattered by a small macroscopic object like an atomic cloud in our case, which has a spatial scale around $1 \mathrm{~mm}$ or less, can be accumulated and focused in a small image spot with an ideal optical system and then detected, see Ref.[14]. In the linear optics, operating with mirrors, lenses etc., the image created by the retrieved signal pulse, would have a spatial profile and polarization, which would be unique for each particular mode of the incoming signal pulse but differ from it. Evidently there are some losses of the pulse intensity in this process and additional noise light, coming from other scattering channels, could contribute to the observation channel. We anticipate that, for linear optics in some cases, this noise can be eliminated in the observation channel after a round of optimization. In this sense we would like to point out one recent proposal of the effective and noiseless conversion of a plane to spherical wave with parabolic mirror [15].

In an alternate possibility to be explored, recovery of the scattered light over a large solid angle may be overcome by applying a time reversal approach. The spin coherence, which is created by diffusely propagating light, reveals a so-called quantum hologram of the scattered light. If the hologram was transformed via a non-linear complex conjugate operation, then one could recover the object/signal pulse in its original profile, i.e. its spatial mode and polarization. Approximately this could be done if we set the entire process to evolve in the spatial area bounded by a non-linearly reflecting medium known as a phase conjugate mirror, see [14], the outgoing signal light would be a time reversed copy of the incident pulse. However for such a mirror there is always extra radiation (noise) created by the mirror itself. In the ideal conditions the expected relation between the average photon number in the outgoing pulse and the similar photon number in the incoming pulse is given by $\bar{n}^{\text {(out) }}=\bar{n}^{\text {(in) }}+1$. The additional photon arrives because of noise and it cannot be eliminated in the observation channel via any optimization procedure.

In the single photon quantum memory protocol both the observation schemes can be considered as equivalent to a beamsplitter, which transmission efficiency $\eta$ is equal to the overall efficiency for write-in and readout stages of the protocol and also includes technical losses of the detection channel, see Figure 4. The noise, associated with other possible scattering channels or radiation sources, can be introduced in this scheme via sending the thermal light onto the second input port of the beamsplitter, such that it would generate the Gaussian (also thermal) light with average number of photons $\bar{n}$ in the observation channel. The observation channel in this model is performed by the output port of the beamsplitter, 


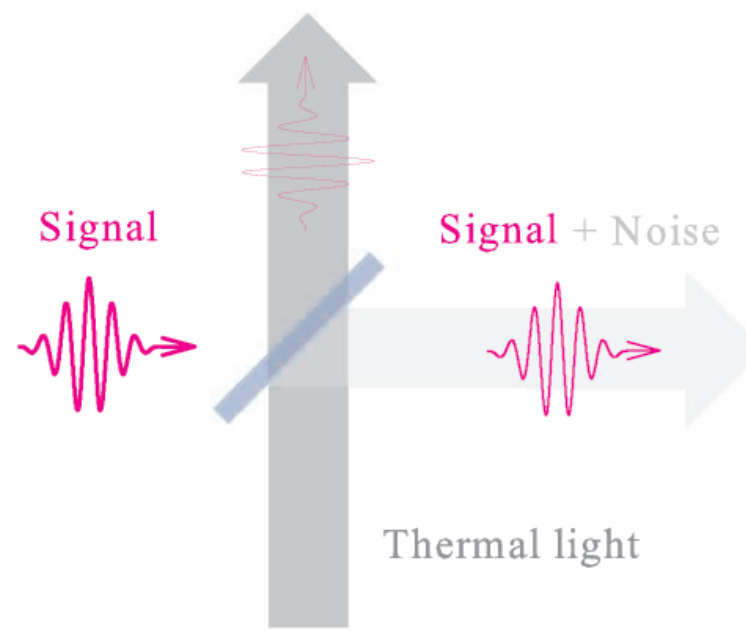

Fig. 4. (Color online) Equivalent scheme for the quantum memory channel. The signal single photon pulse passes through a beamsplitter, with transmittance equal to the efficiency of the memory channel. The additional noise is admixed via sending the thermal light onto the second port of the beamsplitter.

see Figure 4. The single photon input state is described by the following Wigner function

$$
W^{(1)}(\alpha)=\frac{8}{\pi}\left[|\alpha|^{2}-\frac{1}{4}\right] \mathrm{e}^{-2|\alpha|^{2}}
$$

where $\alpha=x+i p$ is the complex field amplitude, and $x$ and $p$ are the quadrature components of the signal mode. After mixing with the thermal light the modified Wigner function in the observation channel is given by

$$
W(\alpha)=\frac{4}{\pi(\bar{n}+1 / 2)}\left[\frac{\eta|\alpha|^{2}}{4(\bar{n}+1 / 2)^{2}}-\frac{1}{4}+\frac{\bar{n}+(1-\eta) / 2}{2(\bar{n}+1 / 2)}\right] \exp \left[-\frac{|\alpha|^{2}}{\bar{n}+1 / 2}\right]
$$

and includes losses with efficiency $\eta<1$ and admixture of thermal noise with average number of thermal photon per mode $\bar{n}>0$. This Wigner function generally describes many photon states and has non-vanishing overlap with the vacuum state as well.

Let us simplify the expression (77) and consider it in two limiting situations: first, for a lossless channel $(\eta=1)$ but with noise; and second, with losses but without noise $(\bar{n}=0)$. For the lossless configuration the Wigner function (7) describes the quantum state where the number of photons per mode, which could be randomly detected in the observation channel, runs the sequence $n=0,1,2, \ldots$. If we post-selected only single photon events with $n=1$, then the probability to observe either signal or noise photon would depend on $\bar{n}$ and $\bar{n}=1$ determines the balance situation, when these probabilities are equal. This just relates to the 
ideal observation conditions based on the phase conjugate mirror technique. If the quantum information was encoded in the polarization state of the signal photon, when the quantum statistical ensemble for the polarization state of the retrieved photon would be described by the following Werner-type density matrix [8, 16]

$$
\hat{\rho}=x|\psi\rangle\left\langle|\psi|+\frac{1-x}{2} \hat{I}\right.
$$

where $|\psi\rangle$ is the original polarization state and $\hat{I}$ is a unit matrix in two dimensional Hilbert space. For $\bar{n}=1$ the weighting parameter $x=1 / 2$ means equal admixture of detecting events for either informative or noise photons. In this case the achieved fidelity $F=\langle\psi|\hat{\rho}| \psi\rangle=3 / 4$ is higher than classical benchmark based on single measurement $F_{\text {meas }}=2 / 3$, see Ref.[17], but less than optimal cloning benchmark $F_{\text {clon }}=5 / 6$, see Ref. [8]. Evidently, to improve the quality of the memory channel a certain distillation procedure is needed, see Refs. [1, 2], and we skip discussion of this subtle problem here.

In the case of a noiseless channel $(\bar{n}=0)$ the Wigner function (7) performs the superposition of the single photon (with weight $\eta$ ) and vacuum (with weight $1-\eta$ ) states of light. The post-selection of the single photon outcome with probability $\eta$ makes a resource for the conditional quantum memory scheme based on the consequence that each successful single photon detection always gives us the informative photon. However, as was pointed out above, this could be reliably done for detection of the retrieved photon with the set of linear optical devices such that the original spatial mode and polarization state of the signal photon would be unpredictably transformed by the scattering channel. Then it might seem that such quantum memory would be useless for the quantum information implication. But we shall show one example where such a memory scheme could be applicable and could enhance reliability of quantum network.

In Figure 5(a) we reproduce the standard applied conditional Bell-type projection measurement for a photon pair, considered as information carrier, with a beamsplitter, see [8]. In this measurement if both the photons have arrived to the input ports of the beamsplitter simultaneously then the detectors' clicks on each of two output ports tells us that the photon state was projected onto the following antisymmetric polarization state

$$
|\Psi\rangle=\frac{1}{\sqrt{2}}[|\uparrow, \leftrightarrow\rangle-|\leftrightarrow, \uparrow,\rangle]
$$

where for the sake of concreteness we expanded this state in the basis of linear polarizations. This can be also called as anti-bunching effect of the photon pair passed through the 
(a)
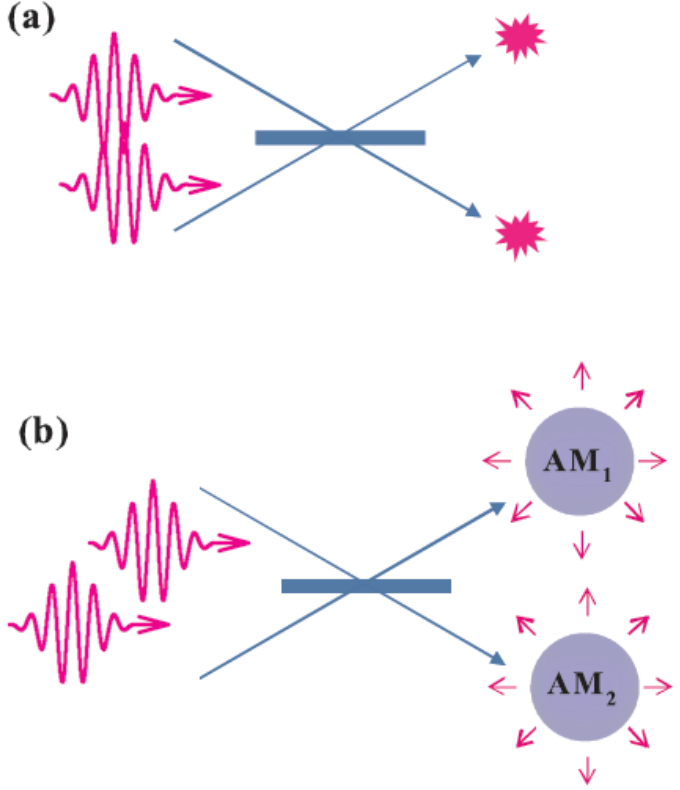

Fig. 5. (Color online) Bell-type measurement based on anti-bunching of the photon pair passed through a beamsplitter. In the standard measurement scheme (a) both the photons should arrive to the input ports simultaneously. With the quantum memories (b) the measurement can be postponed such that the photons could arrive at any time.

beamsplitter.

The technical problem with such measurement is that the coherence volumes of the incoming photons should be essentially overlapped i. e. the coincidence in their arrival time is a crucial requirement for the measurement. If we set the quantum memories in the output, how it is shown in Figure [5(b), this inconvenience could be overcome. Indeed, the discussed memory protocol would allow us to subsequently share the unknown state of each photon between two atomic memory units $\mathrm{AM}_{1}$ and $\mathrm{AM}_{2}$. Then at the readout stage both the photons could be released with much weaker control field than that was used in the write-in stage. This operation leads to essential extension of the outgoing pulse duration. As a consequence, the wavepackets of the released photons would be overlapped and the photons would be indistinguishable such that their further detection would give us the same projection measurements onto the state (9) as in the standard scheme. This detection scheme would be insensitive to the spatial and polarization modes of the outgoing photons and in case of general quantum network consisted of many nodes the proposed integrated architecture of the Bell detector and quantum memories could enhance the network reliability. 


\section{Conclusion}

In conclusion, we have shown that a disordered atomic medium, which diffusely scatters the light propagating through it, can be used as a memory unit for light storage. The mechanism of the Raman-type conversion of the signal pulse into the spin coherence is more effective in this case and the level of losses is much smaller than for the reference process controlling only the forwardly propagating part of the light.

Such effective light storage can be integrated into a quantum network in combination with the standard conditional two-photon Bell detector. Bell detection performs an anti-bunching of the photons overlapped with a beamsplitter and then detected. The main advantage of the proposed scheme is that with the quantum memories the anti-bunching of the detected photons can be observed, even if the photons arrive to the input ports of the beamsplitter at different times.

\section{Acknowledgments}

This work was supported by the Russian Foundation for Basic Research (Grant 10-02-00103 and the National Science Foundation (NSF-PHY-0654226). L.G. acknowledges financial support from the charity foundation Dynasty.

\section{Appendix A: The nonlinear susceptibility and scattering tensor}

The susceptibility tensor responsible for elastic and mesoscopically averaged propagation of the signal mode, or of any its scattered fragments, through the atomic sample can be introduced in its major reference frame. For the excitation geometry shown in Figure 1 where the control mode is linearly polarized along the $Z$-axis it has the following diagonal form

$$
\hat{\chi}=\left(\begin{array}{ccc}
\chi_{\perp} & 0 & 0 \\
0 & \chi_{\perp} & 0 \\
0 & 0 & \chi_{\|}
\end{array}\right)
$$

where the $\chi_{\|}$component determines the response of the atomic polarization on its excitation by the probe mode linearly polarized along the direction of the $Z$-axis and the transverse component $\chi_{\perp}$ gives the response of the probe for any polarization in the $X, Y$-plane. As an example, in Figure 6 we show the excitation diagram of ${ }^{85} \mathrm{Rb}$ when the polarization vector of the signal mode is orthogonal to the polarization vector of the control field. 


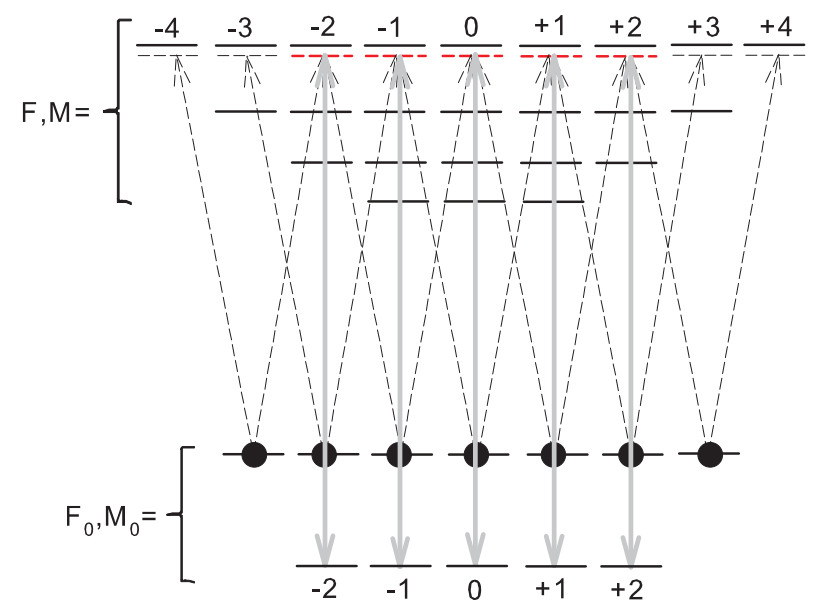

Fig. 6. (Color online) The scheme of excitation of ${ }^{85} \mathrm{Rb}$, when the signal mode (dashed arrow lines) is orthogonally polarized to the control mode (gray arrow lines). The control mode is linearly polarized and the quantization axis $Z$ is directed along its polarization, see Figures 1 and 3 (a).

For an ensemble of motionless atomic scatterers distributed in space with density $n_{0}(\mathbf{r})$ the principal components of the susceptibility tensor are given by

$$
\chi_{\mathbf{j}}(\mathbf{r} ; \omega)=-n_{0}(\mathbf{r}) \frac{1}{2 F_{0}+1} \sum_{m} \sum_{n, n^{\prime}} \frac{1}{\hbar}\left(\mathbf{d e}_{\mathbf{j}}\right)_{n m}^{*}\left(\mathbf{d e}_{\mathbf{j}}\right)_{n^{\prime} m} G_{n n^{\prime}}^{(--)}\left(\hbar \omega+E_{m}+i 0\right)
$$

where $\mathrm{j}=X, Y(\perp) ; Z(\|)$ and $\left(\mathbf{d e}_{\mathbf{j}}\right)_{n m}$ are the matrix elements between the ground $(|m\rangle)$ and excited $\left(|n\rangle\right.$ and $\left.\left|n^{\prime}\right\rangle\right)$ states for the atomic dipole operator projected on the major axis directions. The ground state Zeeman sublevels are specified by the quantum numbers $m \equiv F_{0}, M_{0}$, where $F_{0}=F_{+} \equiv I+1 / 2$, is the total (electronic $S=1 / 2$ and nuclear $I$ ) angular momentum of the upper hyperfine sublevel and $M_{0}$ is its projection. Each Zeeman sublevel has energy $E_{m}$ and all of them are assumed to be equally populated. The quantum numbers $n \equiv F, M$ and $n^{\prime} \equiv F^{\prime}, M^{\prime}$ belong to the set of those Zeeman states of the hyperfine sublevels $F=I+3 / 2, \ldots, I-3 / 2$ of the excited state in $D_{2}$ line, which are accessible by excitation for the signal mode, see excitation diagram shown in figure 6.

The most important ingredient contributing to Eq. (A2) is the retarded-type atomic Green's function of the excited state $G_{n n^{\prime}}^{(--)}(E)$. For the upper exited state $|n\rangle$ with $F=F_{\max } \equiv I+3 / 2$, which is not disturbed by the control mode, this function is simply defined

$$
G_{n n^{\prime}}^{(--)}(E)=\delta_{n n^{\prime}} \frac{\hbar}{E-E_{n}+i \hbar \gamma / 2}
$$


where $E_{n}$ is the energy of the state and $\gamma$ is its natural relaxation rate. The Green's functions for two other contributing states $|n\rangle,\left|n^{\prime}\right\rangle$ form a matrix block of $2 \times 2$ (with $F=$ $I+1 / 2, I-1 / 2$ and with same $M)$. However, these atomic resonances are strongly affected by the interaction with the control mode such the full set of the coupled master equations generally includes a block of $3 \times 3$ matrix components $G_{n n^{\prime}}^{(--)}(E)$ (with $F=I+1 / 2, I-1 / 2$ and additionally $F=I-3 / 2$ all with same $M$, see Figure 6) and this block of equations is given by

$$
\sum_{n^{\prime \prime}}\left[\left(E-E_{n}+i \hbar \frac{\gamma}{2}\right) \delta_{n n^{\prime \prime}}-\frac{V_{n m^{\prime}} V_{n^{\prime \prime} m^{\prime}}^{*}}{E-\hbar \omega_{c}-E_{m^{\prime}}}\right] G_{n^{\prime \prime} n^{\prime}}^{(--)}(E)=\hbar \delta_{n n^{\prime}}
$$

where $V_{n m^{\prime}}$ and $V_{n^{\prime \prime} m^{\prime}}$ are the matrix elements of interaction with the control mode. It is important that for each particular block of states $|n\rangle,\left|n^{\prime}\right\rangle$ and $\left|n^{\prime \prime}\right\rangle$ there is only one Zeeman sublevel in the ground state $\left|m^{\prime}\right\rangle \equiv\left(F_{0}=F_{-}=I-1 / 2, M_{0}=M\right)$, which is coupled with them. The same is also valid for the populated state $|m\rangle$, if one reconsiders the problem in the basis of circular polarizations in the $(X, Y)$-plane, as is shown in Figure 6. This basis is given by the following alternative complete set of unit vectors

$$
\mathbf{e}_{0}=\mathbf{e}_{Z}, \quad \mathbf{e}_{ \pm 1}=\mp \frac{\mathbf{e}_{X} \pm i \mathbf{e}_{Y}}{\sqrt{2}}
$$

and it was used in our calculations. Thus for each particular block of states $|n\rangle,\left|n^{\prime}\right\rangle$ and $\left|n^{\prime \prime}\right\rangle$ there is only one pair of the $\Lambda$-coupled Zeeman sublevels $|m\rangle$ and $\left|m^{\prime}\right\rangle$ in the ground state and each block of equations (A4) is fully specified by only one state $|m\rangle$.

The susceptibility tensor in its major frame can be subsequently built via analytical solution of equations (A4) for each $m$ and next by substituting the result into expression (A2). Then the tensor's components in an arbitrary frame can be found via general rotational transformation. The "dressing" of the atoms by the control mode creates a quasi-energy resonance structure, which is known as Autler-Townes (AT) effect, see Refs. [12, 13], and the location of the AT-resonances is shown by dashed red bars in Figure 6. Because of the AT effect the susceptibility tensor becomes anisotropic and can be naturally divided into linear isotropic and nonlinear anisotropic parts as is shown by Eq.(2). The propagation of the signal pulse or its scattered fragment in any direction can be described by the standard approach of macroscopic Maxwell theory extended by the Green's function formalism, similar to how it was done in [18].

Any probe wave impinging a medium and originally freely propagating in it will be scattered by atomic dipoles. The scattering process in the medium is conveniently described 
by the scattering tensor formalism, see Ref.[19]. This tensor is responsible for frequencyand polarization-dependent transformation of an incident electromagnetic plane wave as a result of its scattering on an isolated atom. The scattering tensor is given by

$$
\begin{aligned}
& \hat{\alpha}_{p q}^{\left(m^{\prime \prime} m\right)}(\omega) \equiv \alpha_{p q}^{\left(m^{\prime \prime} m\right)}(\omega)\left|m^{\prime \prime}\right\rangle\langle m| \\
& \quad=-\sum_{n, n^{\prime}} \frac{1}{\hbar}\left(d_{p}\right)_{m^{\prime \prime} n}\left(d_{q}\right)_{n^{\prime} m} G_{n n^{\prime}}^{(--)}\left(\hbar \omega+E_{m}+i 0\right)\left|m^{\prime \prime}\right\rangle\langle m|
\end{aligned}
$$

where the tensor indices defined either in a Cartesian basis $p, q=x, y, z$ or in a complex angular momentum basis $p, q=0, \pm 1$ for the atomic dipole operators can be confined with any reference frame. The most important difference between expressions (A2) and (A6) is that in the scattering tensor the output transition is open for any accessible atomic state $\left|m^{\prime \prime}\right\rangle$, i. e. the entire scattering process includes all the elastic Rayleigh, elastic Raman, and inelastic Raman scattering channels.

\section{References}

1. E. Polzik, A. Sørensen, K. Hammerer, "Quantum interface between light and atomic ensembles," Rev. Mod. Phys. 82, 1041-1093 (2010).

2. C. Simon et al., "Quantum Memories," Eur. Phys. J. D 58, 1 (2010).

3. R. Zhao, Y. O. Dudin, S. D. Jenkins, C. J. Campbell, D. N. Matsukevich, T. A. B. Kennedy, and A. Kuzmich, "Long-lived quantum memory," Nature Physics 5 100-104 DOI: 10.1038/NPHYS1152 (2008).

4. I. Novikova, A. V. Gorshkov, D. F. Phillips, A. S. Sørensen, M.D. Lukin, and R. L. Walsworth, "Optimal control of light pulse storage and retrieval," Phys. Rev. Lett. 98, 243602 (2007).

5. K. S. Choi, H. Deng, J. Laurat, and H. J. Kimble, "Mapping photonic entanglement into and out of a quantum memory," Nature 452, 67 (2008).

6. A. Amari, A. Walther, M. Sabooni, M. Huang, S. Kröll, M. Afzelius, I. Usmani, B. Lauritzen, N. Sangouard, H. de Riedmatten, N.Gisin, "Towards an efficient atomic frequency comb quantum memory," arXiv:0911.2145v1 (2009).

7. H.-J. Briegel, W. Dür, J.I. Cirac, and P. Zoller, "Quantum repeaters for communication," Phys. Rev. Lett. 81, 5932 (1998). 
8. D. Bouwmeester, A. Ekkert, A. Zeilinger The Physics of Quantum Information (Springer-Verlag Berlin Heidelberg, 2000).

9. N. Sangouard, C. Simon, N. Gisin, J. Laurat, R. Tualle-Brouri, P. Grangier, "Quantum repeaters with entangled coherent states," arXiv:quant-ph/0912.3871v1 (2009).

10. J.B. Brask, I. Rigas, E.S. Polzik, U.L. Andersen, A.S. Sørensen, "A Hybrid LongDistance Entanglement Distribution Protocol," arXiv:quant-ph/1004.0083v1 (2010).

11. D.V. Kupriyanov, I.M. Sokolov, C.I. Sukenik, and M.D. Havey, "Coherent backscattering of light from ultra cold and optically dense atomic ensembles," Laser Phys. Lett. 3, 223-243 (2006); Robin Kaiser, "Quantum Multiple Scattering," J. Mod. Optics 56, 2082 (2009); G. Labeyrie, "Coherent Transport of Light in Cold Atoms," Mod. Phys. Lett. B 22, 73 (2008).

12. S.H. Autler, C.H. Townes, "Stark effect in rapidly varying fields," Phys.Rev. 100, 703-722 (1955).

13. V.S. Letokhov, V.P. Chebotaev Nonlinear laser spectroscopy (Springer-Verlag, Berlin, 1977).

14. M. Born and E. Wolf Principle of Optics (Pergamon Press, Oxford, 1964); L. Mandel and E. Wolf Optical Coherence and Quantum Optics (Cambridge University Press, 1995).

15. M. Sondermann, R. Maiwald, H. Konermann, N. Lindlein, U. Peschel, and G. Leuchs, "Design of a mode converter for efficient light-atom coupling in free space," Appl. Phys. B, 89, 489-492 (2007); R. Maiwald, G. Leuchs, D. Leibfried, J. Britton, J. C. Bergquist, and D. J. Wineland, "Ion traps with enhanced optical and physical access" arXiv:quant-ph/0810.2647v2 (2009).

16. R.F. Werner, "Quantum states with Einstein-Podolsky-Rosen correlations admitting a hidden-variable model," Phys. Rev. A 40, 8, 4277-4281 (1989).

17. S. Massar and S. Popescu, "Optimal Extraction of Information from Finite Quantum Ensembles," Phys. Rev. Lett. 74, 1259-1263 (1995).

18. V.M. Datsyuk, I.M. Sokolov, D.V. Kupriyanov, and M.D. Havey, "Diffuse light scattering dynamics under conditions of electromagnetically induced transparency," Phys. Rev. A 74, 043812 (2006); V.M. Datsyuk, I.M. Sokolov, D.V. Kupriyanov, and M.D. Havey, "Electromagnetically induced optical anisotropy of an ultracold atomic medium," ibid 77, 033823 (2008); I.M. Sokolov, D.V. Kupriyanov, R.G. Olave, and 
M.D. Havey, "Light trapping in high density ultracold atomic gases for quantum memory applications," J. Mod. Optics, 57, 1833 (2010).

19. V. B. Beresteskii, E. M. Lifshits, and L. P. Pitaevskii, Course of Theoretical Physics: Quantum Electrodynamics (Pergamon Press, Oxford, 1981). 